\title{
The optomotor response of aging zebrafish reveals a complex relationship between visual motion characteristics and cholinergic system
}

\author{
Aysenur Karaduman a,b,c, Elif Tugce Karoglu-Eravsar ${ }^{\mathrm{b}, c, d}$, Utku Kaya ${ }^{\mathrm{a}, 1}$, Alaz Aydin ${ }^{\mathrm{a}}$, \\ Michelle M. Adams $s^{\mathrm{a}, \mathrm{b}, \mathrm{c}, \mathrm{d}, \mathrm{e}}$, Hulusi Kafaligonul ${ }^{\mathrm{a}, \mathrm{b}, \mathrm{c}, \mathrm{d}, *}$ \\ a National Magnetic Resonance Research Center (UMRAM), Bilkent University, Ankara, Turkey \\ ${ }^{\mathrm{b}}$ Interdisciplinary Neuroscience Program, Aysel Sabuncu Brain Research Center, Bilkent University, Ankara, Turkey \\ ${ }^{\mathrm{c}}$ Department of Molecular Biology and Genetics Zebrafish Facility, Bilkent University, Ankara, Turkey \\ ${ }^{\mathrm{d}}$ National Nanotechnology Research Center (UNAM), Bilkent University, Ankara, Turkey \\ e Department of Psychology, Bilkent University, Ankara, Turkey
}

\section{A R T I C L E I N F O}

\section{Article history:}

Received 16 March 2020

Received in revised form 10 October 2020

Accepted 17 October 2020

Available online 23 October 2020

\section{Keywords:}

Aging

Motion detection

Direction

Zebrafish

Acetylcholine

\begin{abstract}
A B S T R A C T
Understanding the principles underlying age-related changes in motion perception is paramount for improving the quality of life and health of older adults. However, the mechanisms underlying age-related alterations in this aspect of vision, which is essential for survival in a dynamic world, still remain unclear. Using optomotor responses to drifting gratings, we investigated age-related changes in motion detection of adult zebrafish (wild-type/AB-strain and ache $e^{\text {sb55/+ }}$ mutants with decreased levels of acetylcholinesterase). Our results pointed out negative optomotor responses that significantly depend on the spatial frequency and contrast level of stimulation, providing supporting evidence for the visual motion-driven aspect of this behavior mainly exhibited by adult zebrafish. Although there were no significant main effects of age and genotype, we found a significant three-way interaction between contrast level, age, and genotype. In the contrast domain, the changes in optomotor responses and thus in the detection of motion direction were age- and genotype-specific. Accordingly, these behavioral findings suggest a strong but complicated relationship between visual motion characteristics and the cholinergic system during neural aging.
\end{abstract}

(C) 2020 Elsevier Inc. All rights reserved.

\section{Introduction}

Healthy aging is notably accompanied by changes in visual functioning. These changes severely influence daily activities and have been associated with several dangerous situations such as motor vehicle crashes (Langford and Koppel, 2006; Owsley et al., 1998) and increased risk of falls due to troubles with balance (Lord et al., 2010). Accordingly, these life-threatening problems highlight the importance of characterizing age-related changes in visual functioning and further understanding of the underlying neural mechanisms (Owsley et al., 2016).

Given that motion perception is essential to survival in a dynamic world, most of the previous studies on deficits associated with the aging visual system have particularly focused on motion. Psychophysical studies revealed deficits in motion detection,

\footnotetext{
* Corresponding author at: Aysel Sabuncu Brain Research Center Bilkent University, Ankara 06800, Turkey. Tel.: +90 312290 3016; fax: +90 3122903001 .

E-mail address: hulusi@bilkent.edu.tr (H. Kafaligonul).

1 Department of Anesthesiology, University of Michigan, Ann Arbor, MI.
}

direction, and speed discrimination in older adults (Ball and Sekuler, 1986; Bennett et al., 2007; Norman et al., 2003; Raghuram et al., 2005; Snowden and Kavanagh, 2006). A growing body of literature suggests that age-related deficits in visual motion perception are mainly due to the alterations at low- and mid-level cortical processing rather than those at the sensory organ stage such as optical or retinal problems (Betts et al., 2009; Schmolesky et al., 2000; Yang et al., 2008; Zhang et al., 2008). Previous neuroimaging studies on humans have shown that a decrease in lowlevel radial motion processing with aging is accompanied by the increased activation in the temporal and frontal areas (i.e., middle temporal gyrus and inferior frontal gyrus) of older adults, which was interpreted as a possible compensatory mechanism for the deteriorated perceptual performance (Biehl et al., 2017). The hemodynamic response over the middle temporal area (V5/MT, a key neural substrate of visual motion processing; see Born and Bradley, 2005; Zeki, 2015 for reviews) was found to be greater for older adults who had decreased performance in global motion perception (Ward et al., 2018). These studies overall support the view that 
additional compensatory cortical recruitment is needed to process motion information in older individuals. Moreover, invasive neurophysiological recordings in the visual cortex of old macaque monkeys, without damaged optic nerve or retinal pathology, have shown that the cells in primary visual cortex (V1) and area MT, had lower contrast sensitivity, decreased orientation-, direction-, and speed-selectivity, increased spontaneous activity and decreased signal to noise ratio (Liang et al., 2010; Schmolesky et al., 2000; Wang et al., 2014; Yang et al., 2008, 2009a; Zhang et al., 2008). Moreover, the declines in optimal spatial and temporal frequencies of V1 cells and an overall decrease in preferred speed of MT cells have also been reported (Yang et al., 2009b; Zhang et al., 2008). Functional deterioration in visual motion processing without major changes in neuron number, size, or density in the retino-geniculostriate pathway (Ahmad and Spear, 1993; Kim et al., 1997; Peters et al., 1997; Peters and Sethares, 1993; Vincent et al., 1989) also suggest that there may be, rather, more subtle alterations in synaptic structure or composition, such as changes in synaptic protein levels, likely to contribute to changes in perceptual processes in the aging brain as it was shown for cognitive processing previously (Adams et al., 2008; Clayton et al., 2002; Morrison and Hof, 1997). However, within the context of visual motion, the functional links between age-related changes in behavior and key synaptic targets are still poorly understood.

Zebrafish (Danio rerio) have become an appealing model for studying both age-related behavioral and biological changes. They are inexpensive to maintain, very fertile, and develop extremely rapidly, becoming sexually mature in 3 months. Their brain and nervous system develop similarly compared with those of other vertebrates (Kimmel, 1993). Ease of genetic manipulation, high degree of similarity between zebrafish and human genes, the availability of multiple mutant and transgenic phenotypes, and identified biomarkers of aging make zebrafish a highly valuable model for genetic analyses and biological aging processes (Arslan-Ergul et al., 2016; Gerhard, 2003; Howe et al., 2013; Kalueff et al., 2014; Kishi et al., 2008). More importantly, as in humans, zebrafish exhibit age-related declines in cognitive functions (e.g., learning and memory), and accumulating evidence suggests that subtle changes in cellular and synaptic integrity underlie these changes (Adams and Kafaligonul, 2018). Zebrafish offer various advantages as well for studying vision. Their visual system is similar to those of other vertebrates in terms of retinal anatomy, the organization of retinotectal projections and pathways (Bilotta and Saszik, 2001; Schmitt and Dowling, 1999). In addition, there are several procedures available to quantify a wide range of stimulus-driven behaviors of zebrafish such as optomotor responses and optokinetic reflexive eye movements. Previous research revealed qualitatively similar visual acuity and contrast sensitivity functions for zebrafish (Tappeiner et al., 2012). Furthermore, it has been shown that zebrafish perceive first- and second-order motions which mainly engage low- and midlevel motion processing. They also experience various motion illusions commonly used in human studies such as reverse-phi, motion aftereffect, and rotating snakes illusions (Gori et al., 2014; Najafian et al., 2014; Orger et al., 2000). The zebrafish visual system also relies on similar principles underlying motion processing to those commonly found in humans and other vertebrates. Many neurons at different stages of the visual system have selective responses to motion direction (Bollman, 2019). Besides retinal ganglion cells sensitive to motion, previous studies emphasize the importance of direction-selective neurons located in the optic tectum and pretectum for further processing (Wang et al., 2020; Yildizoglu et al., 2020). For instance, the percept of motion aftereffect has been associated with the habituation of optic tectum neurons (PerezSchuster et al., 2016). A combination of whole-brain imaging, behavioral analysis, and modeling also revealed that pretectal circuits process retinal inputs and integrate monocular motion cues which subsequently play a critical role in the generation of command signals for the optomotor response (OMR) (Naumann et al., 2016).

Although motion detection of aging zebrafish has not been systematically investigated yet, previous research on cognitive functioning provides important mutant models. In terms of cognitive aging, an interesting zebrafish mutant line is achesb55/+ mutants. These mutants have impaired acetylcholinesterase function which hydrolyzes and inactivates acetylcholine (ACh), and thus resulting in increased ACh levels (Behra et al., 2002; Soreq and Seidman, 2001). ACh is a cortical neuromodulator that plays important roles in various cognitive functions such as attention, learning, and memory (Everitt and Robbins, 1997; Furey et al., 2000; Hasselmo, 1999; Herrero et al., 2008; Sarter et al., 2005). Furthermore, age-related changes in the cholinergic system (i.e., the loss of ACh levels) have been suggested to contribute to cognitive decline in normal aging (Bartus et al., 1982; Schliebs and Arendt, 2011; Terry and Buccafusco, 2003). To understand the cholinergic involvement in cognitive aging, Yu et al. (2006) used ache $e^{s 55 /+}$ mutants in spatial learning paradigms. Compared with wild-type siblings, age-related spatial learning deficits were delayed in these animals, which is consistent with the hypothesis that ACh levels affect age-related changes in cognitive functioning. These mutants have been considered as a delayed model of cognitive aging. ACh also plays a key role in visual processing and perception. ACh application to V1 neurons of macaque monkeys improves spatial tuning (Roberts et al., 2005), increases responsiveness by decreasing contrast thresholds and enhances response gain, and resulting in better detection of visual stimuli (Disney et al., 2007; Soma et al., 2012). Using other species, it has also been shown that ACh improves orientation, direction selectivity, and contrast sensitivity of V1 neurons. Of particular relevance to the present study, an increase in ACh level leads to improvements in the direction selectivity of MT neurons and hence the detection and discrimination of direction while decreasing noise correlations (Thiele et al., 2012). In addition, increasing the levels of ACh via the cholinesterase inhibitor donepezil in humans improves visual perceptual learning and leads to better motion discrimination of the trained stimuli (Rokem and Silver, 2013, 2010). It has been suggested that such ACh effects on visual processing mimic the effects of attention because attention similarly decreases noise correlations and increases sensitivity in visual cortices (Cohen and Maunsell, 2009; Goard and Dan, 2009; Thiele et al., 2009). Surprisingly, despite the evidence demonstrating the various contributions of the cholinergic system to visual processes, its specific roles in age-related changes in visual motion perception have not been studied extensively. Using genetic variations in the alpha 7 subunits of cholinergic nicotinic receptor (CHRNA7), a recent study investigated the relationship between the cholinergic system in humans and agerelated changes in motion perception (Kunchulia et al., 2019). The behavioral findings suggest that genetic variations are associated with perceptual performance on direction discrimination, but these associations seem to be unrelated to age-related changes. Although this study provides an important step along this direction, it is critical to evaluate the modulations of perceptual performance under a rich repertoire of stimulus features because age-related alterations can also be dependent on specific motion characteristics and parameters (see below).

Using the zebrafish model which offers an ideal framework for both aging and visual motion, we focused on characterizing the detection of motion direction during aging. To generate stimulusdriven optomotor responses, we used sine-wave gratings with varying contrasts $(1 \%-75 \%)$ and spatial frequencies $(0.01-0.8 \mathrm{c} /$ $\mathrm{deg}$ ) and assessed the motion detection of young (7-10 months) and old animals (24-43 months). Previous motion studies were mainly focused on zebrafish larvae (e.g., Orger et al., 2000). To date, there is limited information on motion detection of adult zebrafish 
and no systematic investigation of age-related changes in the detection of motion direction. Specifically, we aimed to fill this important gap in the literature. Although earlier research on humans revealed an overall age-related decline of motion detection and direction discrimination performance, relatively recent studies indicated that motion parameters also play a critical role in agerelated changes and thus emphasize interaction with specific stimulus parameters rather than an overall effect of age (e.g., Conlon et al., 2017; Pilz et al., 2017). Building on these findings, we anticipated on finding an interaction between aging and other factors (i.e., contrast or spatial frequency). More importantly, our design also included young and old ache $e^{s b 55 /+}$ mutants. As mentioned previously, the cholinergic system and ACh levels are significantly involved in both aging and visual motion processing. Therefore, we also predicted a differential effect of genotype (i.e., wild-type vs. achesb55/+ mutants) on age-related changes in the detection of motion direction. In this way, we aimed to provide additional key insights into the functional link between visual motion processing and ACh levels during neural aging.

\section{Methods}

\subsection{Animal husbandry}

A total of 70 adult zebrafish (young: $7-10$ months and old: 24-43 months) were used in this study. The data of 3 fish were excluded from further analyses due to technical problems in video tracking, and 5 fish expressed abnormal behaviors in the testing tank during data collection (see Data processing and quantification). Thus, the data of 28 wild-type ( $\mathrm{AB}$ strain: 16 young and 12 old) and 34 mutant $\left(a c h e^{s b 55 /+}: 19\right.$ young and 15 old $)$ zebrafish were used in the analyses. The mutants were originally obtained from the European Zebrafish Resource Center-Karlsruhe Institute of Technology. All fish were raised in standard conditions and maintained at a constant temperature of $28.5^{\circ} \mathrm{C}$ on a $14: 10$ hours light:dark cycle in the Bilkent University Zebrafish Facility. The fish were fed twice a day with standard fish flakes (Sera, Germany) and once a day with fresh artemia in a recirculating tank system (Tecniplast, Italy). The stocking densities were kept as approximately 10 fish in 4-L tanks, and the fish with the same birthdates were housed in the same tank. Animals were maintained with minimal disturbance to prevent any unnecessary stress. Two fish were taken from the facility system each week and kept together in an 8.5-L holding aquaria (Petstore, Ankara, Turkey) for a week during the experiments (see Behavioral testing procedure). The protocol for the behavioral experiments in this study was in accordance with the international guidelines for the care and use of laboratory animals and approved by the Bilkent University Local Animal Ethics Committee with the following approval date and number: August 5, 2015 and no: 2015/44.

\subsection{Experimental set-up and stimuli}

Our experimental design was based on measuring zebrafish optomotor response, a position-stabilizing reflex to whole-field visual motion (see Data processing and quantification). Accordingly, the behavioral set-up consisted of an elongated test tank located in front of an LCD monitor, a video camera, and a computer to control stimulus presentation and recording (Fig. 1A). The test tank $(4 \times 30 \times 20 \mathrm{~cm})$ was filled with $10 \mathrm{~cm}$ of water. To exclude external visual cues and light, white shields were attached to the

A

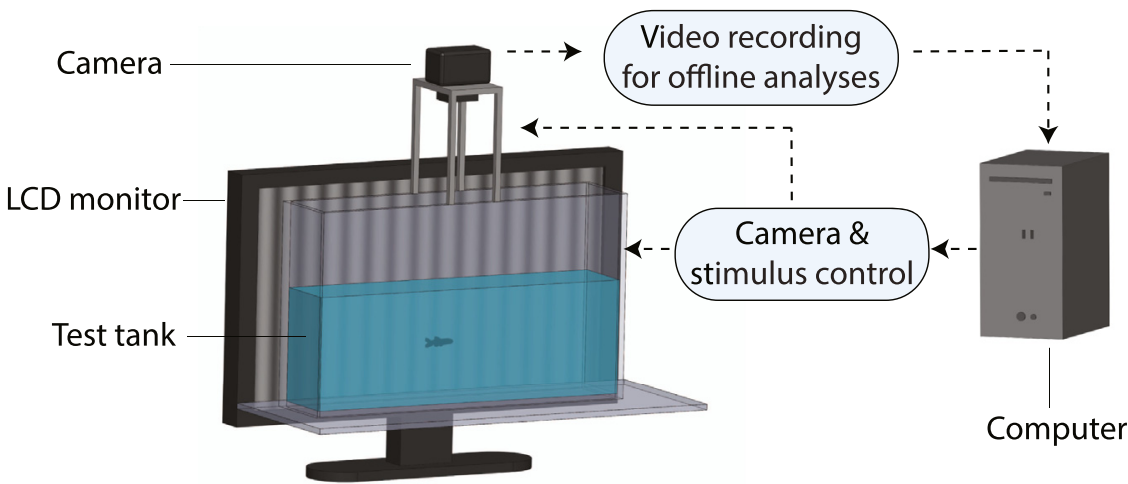

B

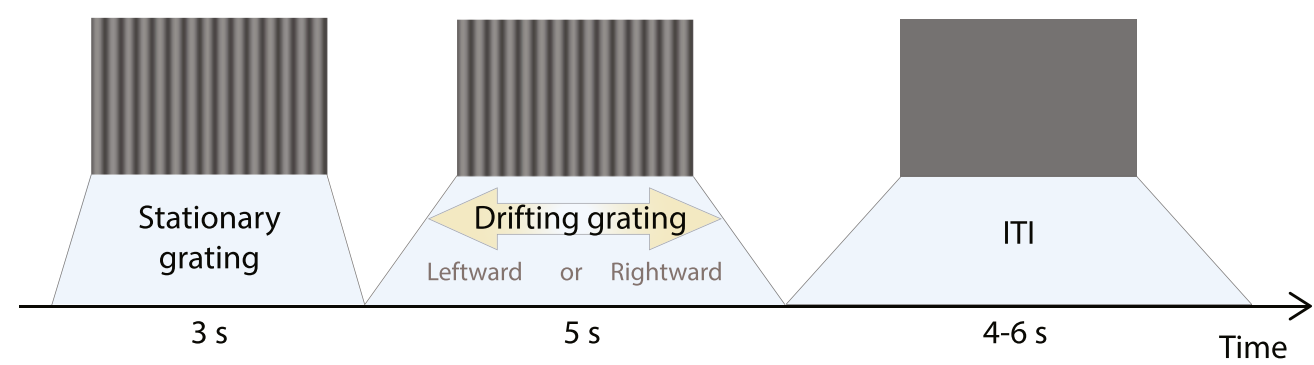

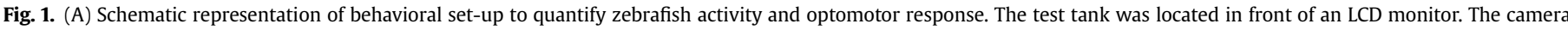

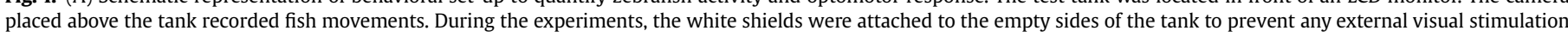

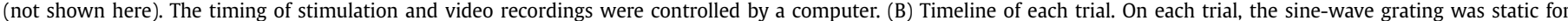

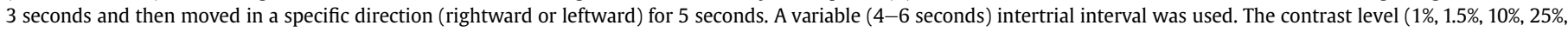
$45 \%$, and $75 \%)$ and spatial frequency $(0.01,0.05,0.1,0.2,0.4$, and $0.8 \mathrm{c} / \mathrm{deg})$ of the grating were varied across trials. 
empty sides. The camera (Logitech HD Pro Webcam C920, 60 frames per second) was placed above the test tank, and it was used to record the behavioral activity of zebrafish for offline analyses. Some preliminary measurements were made using the set-up to optimize the recording settings and position of the camera in terms of image quality. The visual stimuli were presented via an 18.5-inch LCD monitor (HP V196, $1366 \times 768$ pixel resolution and $60 \mathrm{~Hz}$ refresh rate).

A SpectroCAL (Cambridge Research Systems, Rochester, Kent, UK) photometer was used for the luminance calibration and the linearization of the display. The minimum and maximum luminance values of monitor were 0.3 and $175.31 \mathrm{~cd} / \mathrm{m}^{2}$, respectively. The mean luminance of stimulation (i.e., grating and background) was $20 \mathrm{~cd} / \mathrm{m}^{2}$. Stimulus presentation and video recording times were controlled using MATLAB 2016a (The MathWorks, Natick, MA, USA) with Psychtoolbox 3.0 (Brainard, 1997; Pelli, 1997). To generate first-order motion, drifting sine-wave gratings with varying contrasts $(1 \%, 1.5 \%, 10 \%, 25 \%, 45 \%$, and $75 \%)$ and spatial frequencies $(0.01,0.05,0.1,0.2,0.4$, and $0.8 \mathrm{c} / \mathrm{deg}$ ) were used. The range of the contrast levels and spatial frequency values and other stimulus parameters (e.g., speed) was determined as per previous research with zebrafish (Bak-Coleman et al., 2015; Hollbach et al., 2015; Maaswinkel and Li, 2003; Tappeiner et al., 2012). At the beginning of each trial, the drifting grating was static for 3 seconds (Fig. 1B) and then, it drifted in a specific direction (rightward or leftward) for 5 seconds with a speed of $20 \mathrm{deg} / \mathrm{s}$. After a variable (4-6 seconds) intertrial interval, the next trial began. Visual angles were calculated based on a $1.5 \mathrm{~cm}$ distance from the LCD monitor screen.

\subsection{Behavioral testing procedure}

Two zebrafish were taken from the system tanks and transferred to the holding tank in which the fish were held during the testing week. We collected data for 6 different contrast levels at each of the 6 different spatial frequency levels, making 36 conditions in total. These conditions were presented in 6 different sessions such that the gratings with the same contrast level were presented in a single session. In other words, the contrast level of the grating was fixed during each session, and only the spatial frequency was varied across trials. In each session, a grating with a specific contrast and spatial frequency was pseudorandomly presented 30 times (15 times for each motion direction). Thus, each session consisted of 180 trials ( 6 spatial frequencies $\times 30$ trials) and lasted around 40 minutes in total.

Data were collected between 9:30 am-4:00 pm and the measurements lasted 3 days (typically 2 contrast levels/sessions per day) in total. The zebrafish has a light/dark activity cycle such as humans, and this time range corresponds to the active phase of fish (Zhdanova et al., 2008). On each day, each fish was tested separately in the test tank and completed 2 randomly selected contrast levels via 2 consecutive experimental sessions. The order of the testing sessions for the fish was counterbalanced across days. On the first day of the behavioral testing, a habituation session lasting around 40 minutes and consisting of only the highest contrast level grating (75\% contrast level, 6 spatial frequencies $\times 30$ trials) was presented before the main experimental sessions to get the fish acclimated to the testing tank, light conditions, and stimulation. On the following days, we followed the same procedure, but the habituation session was shorter (lasting approximately 20-30 minutes).

\subsection{Genotyping of ache $e^{\text {sb55/+ }}$ mutants}

After the behavioral experiments, the heterozygous ache $e^{s b 5 /+}$ mutants and their wild-type siblings were genotyped. Initially, the
Table 1

q-PCR experiments on the extracted genomic DNAs to distinguish the mutant animals

\begin{tabular}{ll}
\hline q-PCR reactions & Amount $(\mu \mathrm{l})$ \\
\hline Forward primer (N or S, $0.5 \mu \mathrm{M}$ final $)$ & 1 \\
Reverse primer $(0.5 \mu \mathrm{M}$ final $)$ & 1 \\
ddH2O & 2 \\
Genomic DNA (10 $\mu \mathrm{g} / \mu \mathrm{L}$ final $)$ & 1 \\
SYBR Green & 5 \\
\hline
\end{tabular}

zebrafish were euthanized by immersion in ice-water, and then the tails were separated from the body with scissors and snap frozen in liquid nitrogen. The samples were stored at $-80^{\circ} \mathrm{C}$ in $1.5 \mathrm{~mL}$ tubes until the DNA extraction step. Genomic DNA was extracted from the tail samples using standard procedures. For this process, $200 \mu \mathrm{L}$ of lysis buffer containing $100 \mathrm{mM}$ Tris $\mathrm{pH} 8.2$ with $10 \mathrm{mM}$ EDTA, $200 \mathrm{mM} \mathrm{NaCl}, 0.5 \%$ SDS, and $1 \mathrm{X}$ proteinase $\mathrm{K}$ was added to the tail samples, and these were incubated at $55{ }^{\circ} \mathrm{C}$ on a shaking rotates overnight. After this incubation period, proteinase $\mathrm{K}$ was deactivated by heating the samples at $95{ }^{\circ} \mathrm{C}$ for 20 minutes. Then, the samples were centrifuged at $13,000 \mathrm{rpm}$ for 20 minutes at $4{ }^{\circ} \mathrm{C}$. Supernatants were collected into the new tubes, and $175 \mu \mathrm{L}$ of isopropanol was added into them and mixed well. The samples were further centrifuged at $13,000 \mathrm{rpm}$ for 20 minutes at $4{ }^{\circ} \mathrm{C}$, supernatants were carefully discarded, and pellets were washed with $500 \mu \mathrm{L}$ of $70 \%$ ethanol. After this step, the samples were centrifuged one more time at $13,000 \mathrm{rpm}$ for 20 minutes at $4{ }^{\circ} \mathrm{C}$. Supernatants were discarded carefully, and pellets were air-dried for 20 minutes. After this step, the pellets were resuspended in $20 \mu \mathrm{L}$ DNase/RNase-free water and genomic DNA concentrations were determined using NanoDrop (Thermo Scientific, Rockford, IL). Extracted genomic DNAs were subjected to analysis using quantitative polymerase chain reaction (q-PCR) to distinguish the mutant animals (Avci et al., 2018). Two forward primers were used for genotyping (Table 1 ). The first primer, primer $\mathrm{S}$ recognized the wild-type sequence and the second primer $\mathrm{N}$ identified the point mutation existing in heterozygous mutants (Table 2). Each sample was tested with both primers in duplicates, and mutants were determined based on the amplification difference of these 2 primers.

\subsection{Data processing and quantification}

We used OMR to assess motion detection and the perceived direction of adult zebrafish. OMR is a position-stabilizing reflex that includes eye, head, or whole-body movements to whole-field visual motion, and it has been widely used to quantify various visual functions of different species. Through this reflexive behavior, for instance, flying insects can maintain flight control or fish can avoid drifting in water currents and control its orientation by stabilizing the displacement of the visual image on the retina (see Kalueff et al., 2013; Orger and Polevieja, 2017 for reviews). The paradigms based on OMR have been well established for studying visual motion processing in various species. Of particular relevance to the present study, OMR provides reliable behavioral metrics for various aspects of visual motion processing in zebrafish such as first- and second-

Table 2

Primer sequences

\begin{tabular}{lll}
\hline Primer & Forward primer $\left(5^{\prime}-3^{\prime}\right)$ & Reverse primer $\left(3^{\prime}-5^{\prime}\right)$ \\
\hline $\mathrm{S}$ & ACACGTGCCATATTGCAGAG & CTGCTCCAGGGAAGAACTTG \\
$\mathrm{N}$ & ACACGTGCCATATTGCAGAA & \\
\hline
\end{tabular}

S: wild-type sequence; N: mutant sequence (Avci et al., 2018) 
order motion perception (Orger et al., 2000), the spatiotemporal characteristics of motion detection (Maaswinkel and Li, 2003), the contributions of different cone types to motion detection (Krauss and Neumeyer, 2003; Orger and Baier, 2005), and motion aftereffects (Najafian et al., 2014). Compared with other similar reflexive behaviors, the OMR testing procedures provide additional important advantages for studying motion processing in adult zebrafish. For instance, OMR and optokinetic reflexive (OKR) eye movements methods yield similar results and conclusions on rodent vision, such as visual acuity and contrast sensitivity (Kretschmer et al., 2017, 2013; Prusky et al., 2004; Tabata et al., 2010). However, in contrast to OKR, the OMR procedure is easy to apply, and it does not require confinement of the animal, which can be challenging especially for the adult zebrafish (e.g., Tappeiner et al., 2012), thereby allowing the animal to move freely in the testing arena. This is particularly important to minimize stress, especially in old animals, during behavioral testing and to easily identify abnormal behavior at the end.

As in the previous motion studies using a rectangular test tank for OMR paradigm, the position shift of fish along the longer side of the tank was used to quantify OMR. Although zebrafish larvae typically exhibit this shift in the direction of physical motion (positive OMR), previous research also indicated that it can be in the opposite direction (negative OMR) for adult zebrafish (e.g., Bak-Coleman et al., 2015). Accordingly, in each video frame, the position of fish in the test tank was tracked offline via MATLAB Video Processing Toolbox (The MathWorks, Natick, MA, USA) and our own custom scripts. Briefly, the video recordings for each trial were first converted to grayscale, and the average of the whole trial was computed for a representative background model. The region where the fish could be present (i.e., the inside of the test tank) was cropped based on the background model, and then, the background subtraction was applied for each frame. The determinant of Hessian was used for blob detection, and the locations of blob centers in each frame were recorded as horizontal and vertical positions of fish in the test tank (Xu and Cheng, 2017). Finally, a 5th order median filter was applied for smooth movement pattern estimations. To optimize this tracking procedure and to validate the position shift as a behavioral metric of OMR, pilot studies were conducted before starting the main experiment. With a small number of trials during these studies, the drifting gratings elicited reliable and robust position shifts in adult zebrafish. These preliminary results also indicated that the position shifts were mainly in the opposite direction of motion and hence pointed to robust negative OMR for adult zebrafish. Overall, the tracking procedure was highly successful in terms of locating the fish position during both pilot studies and the main experimental sessions (Supplementary Video 1).

During the main experimental sessions, several zebrafish showed specific behaviors that have been associated with stress/ fear or escape response such as the increased speed of movement, diving, rapid directional changes, or freezing (Kalueff et al., 2013). We observed that the frequent display of such behaviors was highly likely to overshadow the true OMR response. Accordingly, before the calculation of OMR, we excluded these trials, sessions, or complete data sets of some fish that had included these behaviors throughout all experimental sessions. One of the most frequent abnormal swimming pattern observed was also swimming in circles at one of the corners while repeatedly diving to the bottom and then back toward the surface (Supplementary Video 2). We detected those behaviors by finding the trials with the mean fish location of less than $5 \mathrm{~cm}$ away from the corners with a standard deviation lower than $3 \mathrm{~cm}$. This method was successful at identifying most of these trials. In rare cases, as we did for other types of abnormal behaviors (e.g., freezing and fast swimming), we manually detected the trials by examining the position coordinates of the fish in the test tank and confirmed such instances by watching the recorded videos in detail. A trial was excluded when any of these abnormal behaviors were present in more than half of the trial. On average, $97.3 \%$ of the trials (SEM $=0.26 \%$ ) were retained per behavioral testing session.

We examined fish swimming patterns within different time windows of 8 seconds of visual stimulation (3 seconds of stationary and 5 seconds of drifting grating) to find the time period which correctly reflects the robust OMR response. These preliminary analyses revealed that the horizontal position of zebrafish (i.e., the elongated axis of the test tank parallel to the drifting stimulation on the display) within the first 3 seconds stimulation of drifting grating reflects reliably the sudden and reflexive response (i.e., OMR) to motion. In addition, because the zebrafish move quickly, after a sudden OMR response to the moving stimulus, they come to the end of the test tank before the stimulus ends and often swim back again. A similar timeline has been recently reported for the OMR of larval zebrafish (Kist and Portugues, 2019). Therefore, we used the average of horizontal position within the first 3 seconds of motion stimulation (i.e., $0-3$ second time window) for further analyses of main experimental sessions. Our preliminary analyses and observations also highlighted the importance of using the last 2 seconds of the stationary period (i.e., -2 to 0 seconds time window) to get rid of any confounding factor due to the startle response to the appearance of grating after the gray background presented between the trials. We used the average position within this time range as the baseline level right before the motion stimulation.

For each zebrafish in an experimental condition, we first referenced the horizontal position values based on the physical motion direction in each trial and the positive and negative values corresponded to a position shift in the same and opposite direction to that of the drifting grating, respectively. These values were then averaged across all trials of each specific condition. This led to an average position estimate of individual zebrafish throughout the presentation of an experimental condition ( 3 seconds of stationary and 5 seconds of drifting grating stimulation). We computed the mean position within the first 3 second time window of drifting grating stimulation and then subtracted the mean position within the 2 second time window right before the onset of motion stimulation (i.e., baseline position level) from this value. Thus, the OMR values of all conditions were calculated in centimeters. Some of the basic locomotor properties (e.g., speed) can affect the raw position shifts in centimeters. For instance, a faster swimming speed can overall lead to larger position shifts within a fixed amount of time (i.e., 3 seconds). In accordance with previous research (e.g., Pix et al., 2000), we used a common normalization procedure to limit the contribution of such potential confounds due to basic locomotor properties. For each zebrafish, the baseline-corrected position shifts were divided by the difference between the maximum and minimum of these values [i.e., (max-min) corresponding to the observed range for an individual fish]. This basic approach allowed us to circumvent potential confounding factors and hence to have a fair comparison across different zebrafish groups. It should be noted that the experimenter was blind to the conditions and groups in all phases of the data analyses.

\subsection{Statistical analysis}

We used normalized OMR values to compute group-averaged OMR responses for each condition and in further statistical tests. The statistical tests were performed with SPSS version 25 (IBM SPSS Statistics, Armonk, NY, USA). Although the data were collected for all the contrast and spatial frequency conditions from each zebrafish, the criteria used in data processing and quantification led to missing data at specific conditions. Therefore, the linear mixed- 
Table 3

One sample t-tests to assess whether the OMR elicited by each condition was significantly different than the baseline zero level. The $p$ values for each contrast level and spatial frequency condition are shown in separate rows and columns, respectively. False discovery rate (FDR) was applied to correct for multiple comparisons. Significant $p$ values $(p<0.05)$ are highlighted in bold

\begin{tabular}{llllrlr}
\hline \multirow{2}{*}{ Contrast (\%) } & \multicolumn{6}{l}{ Spatial frequency (c/deg) } \\
\cline { 2 - 7 } & 0.01 & 0.05 & 0.1 & \multicolumn{1}{l}{0.2} & 0.4 & \multicolumn{1}{c}{0.8} \\
\hline 1 & 0.899 & 0.331 & 0.899 & 0.899 & 0.899 & 0.899 \\
1.5 & 0.899 & 0.805 & 0.805 & 0.899 & 0.604 & 0.824 \\
10 & 0.805 & 0.899 & 0.899 & 0.547 & 0.331 & 0.814 \\
25 & 0.982 & 0.805 & 0.202 & 0.068 & $\mathbf{0 . 0 1 5}$ & 0.805 \\
45 & 0.899 & 0.168 & $\mathbf{0 . 0 1 8}$ & $<\mathbf{0 . 0 0 1}$ & $\mathbf{0 . 0 0 6}$ & 0.098 \\
75 & 0.899 & $\mathbf{0 . 0 3 2}$ & $\mathbf{0 . 0 0 6}$ & $\mathbf{0 . 0 0 6}$ & $\mathbf{0 . 0 0 6}$ & $<\mathbf{0 . 0 0 1}$ \\
\hline
\end{tabular}

effects model procedure was used for the statistical analyses given its efficiency in dealing with missing data (Heck et al., 2014). This statistical procedure has the advantage of allowing for the inclusion of all subjects in the analysis without losing power compared with the standard general linear models and allows combinations of both fixed and random effects (Boisgontier and Cheval, 2016). The model included the main effects and interactions of age, genotype, and repeated measurements of spatial frequency and contrast as fixed effects. The model also had a subject-specific random intercept to account for intraindividual correlation among the measurements collected from a specific fish (Brauer and Curtin, 2018; Schumann et al., 2010). To understand the nature of a main effect or a significant interaction, post hoc simple effect analyses were conducted. Multiple pairwise comparisons were corrected using the false discovery rate procedure (Benjamini and Hochberg, 1995; Benjamini and Yekutieli, 2001). The threshold for significance was set at $p<0.05$.

\section{Results}

Fig. 2 shows the normalized OMR values (i.e., baseline-corrected and normalized position shifts) for each age (young, old) and
A

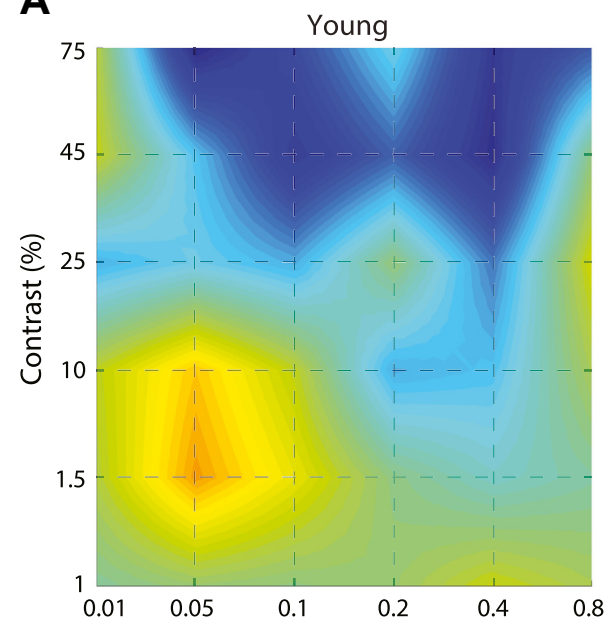

B

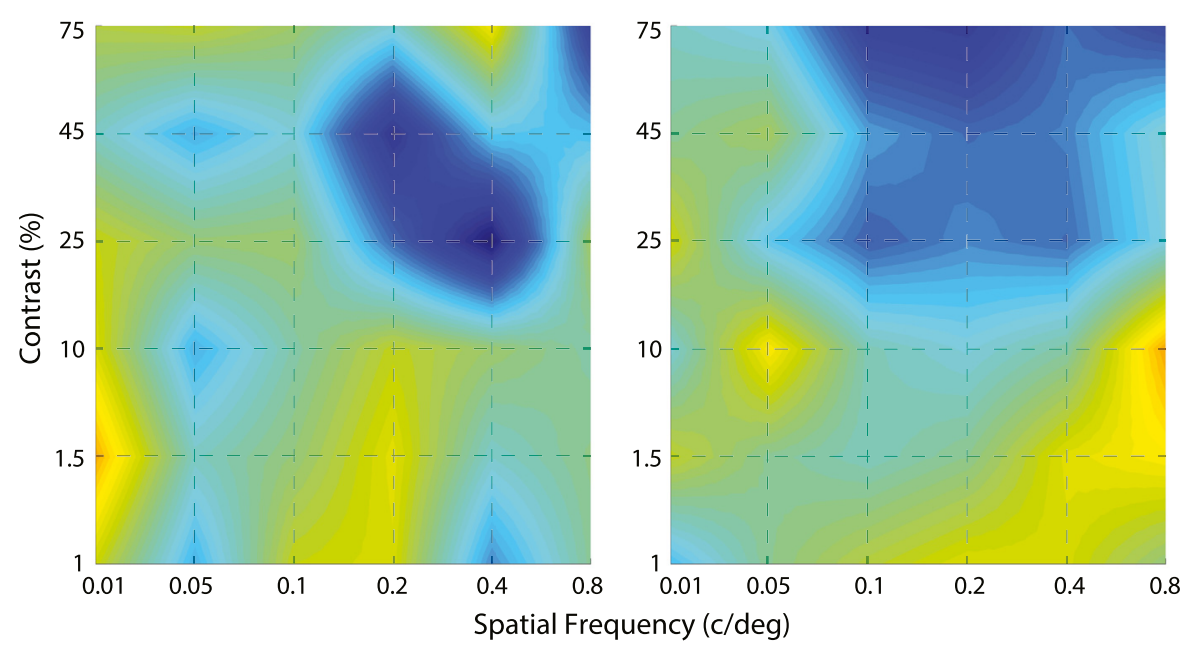

Old
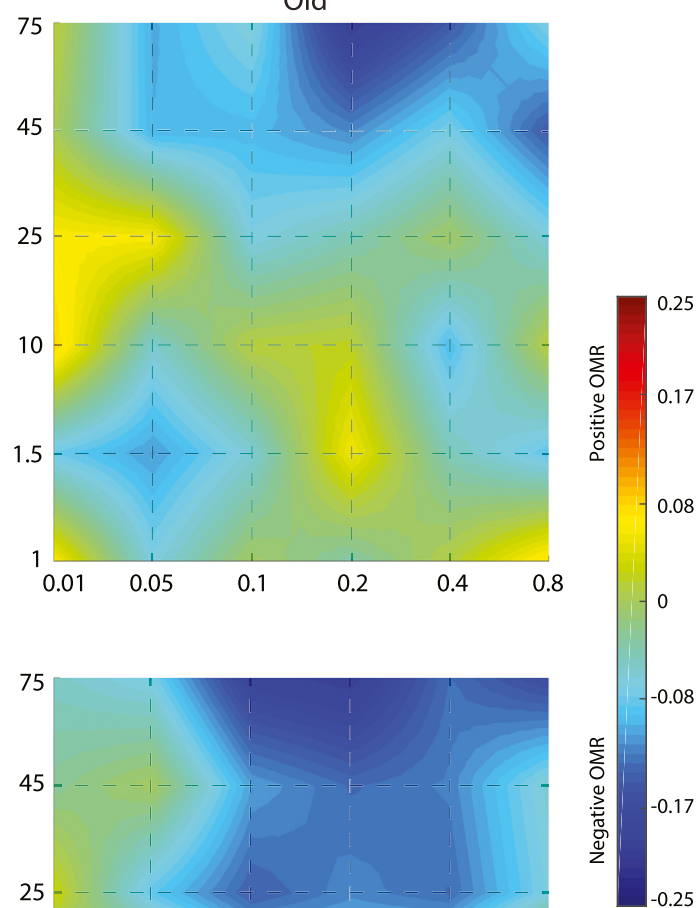

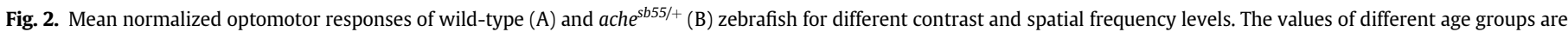

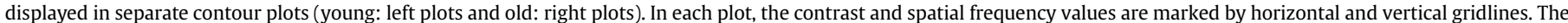

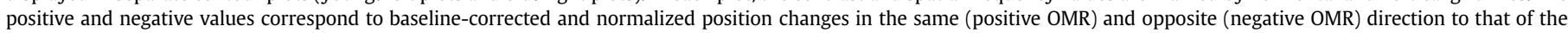

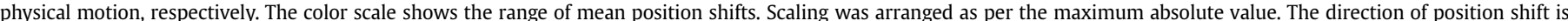

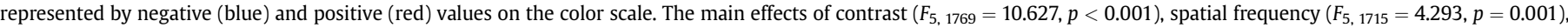

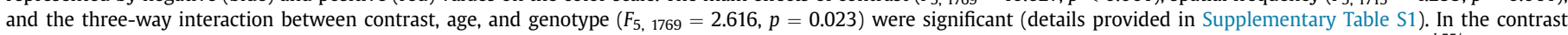

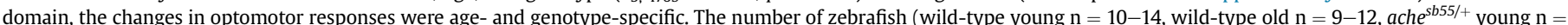

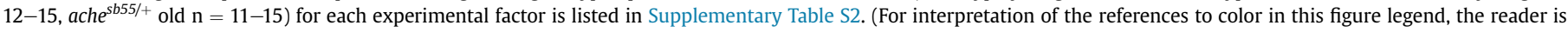
referred to the Web version of this article.) 
genotype (wild-type, $a c h e^{\text {sb55/+ }}$ ) group across 6 contrast levels and 6 spatial frequency conditions. The positive and negative values correspond to position shifts in the same (i.e., positive OMR) and opposite (i.e., negative OMR) direction of physical motion, respectively. As indicated by these contour plots, the negative OMR was dominant beyond the $10 \%-25 \%$ contrast level and $0.05-0.1 \mathrm{c} / \mathrm{deg}$ spatial frequency values. The overall pattern of these negative position shifts also differed for each zebrafish group. That is to say, the optimum combination of contrast and spatial frequency was distinct in terms of normalized magnitudes. This can also be observed in plots displaying OMR values at each contrast level and spatial frequency separately (Supplementary Figure S1 and S2). Typically, adult zebrafish moved up to $6 \mathrm{~cm}$ in the opposite direction at these optimum conditions (Supplementary Figure S3). Notably, the wild-type young and ache $e^{\text {sb55/+ }}$ old groups overall had larger shifts. We first performed a linear mixed-effects model to evaluate these changes in the behavioral measure. The model reported that contrast $\left(F_{5,1769}=10.627, p<0.001\right)$ and spatial frequency $\left(F_{5}, 1715=4.293, p=0.001\right)$ significantly affected the zebrafish OMR to drifting gratings. These statistical results suggest that zebrafish (negative) OMR is significantly dependent on low- level visual motion parameters, and hence highlighting the stimulus-driven nature of this behavior. On the other hand, there was no significant main effect of age $\left(F_{1,58}=0.028, p=0.867\right)$ or genotype $\left(F_{1,58}=0.025, p=0.875\right)$. Interestingly, the linear mixedeffects model indicated only a significant interaction between contrast, age, and genotype $\left(F_{5}, 1769=2.616, p=0.023\right)$. The interaction between spatial frequency, age, and genotype was not significant $\left(F_{5,1715}=1.473, p=0.195\right)$. Moreover, the test did not reveal any other two-way, three-way, or four-way interactions (Supplementary Table S1). In each group, we also compared each stimulation condition with the baseline zero level. These follow-up tests revealed robust negative OMR at the highest 75\% contrast level for wild-type young $(0.05 \mathrm{c} / \mathrm{deg}, p=0.023 ; 0.4 \mathrm{c} / \mathrm{deg}, p=0.026)$ and $a_{c h} e^{s 55 /+}$ old $(0.2 \mathrm{c} / \mathrm{deg}, p=0.005)$ groups. The outcome of these tests is in line with age- and genotype-specific alterations in the contrast domain (i.e., the significant three-way interaction), supporting the original prediction about the differential effects of age and genotype based on visual motion characteristics.

To further understand the dependency of negative OMR on the contrast level and spatial frequency, we combined data across different age and genotype groups (Fig. 3A). The contrast influenced

\section{A}

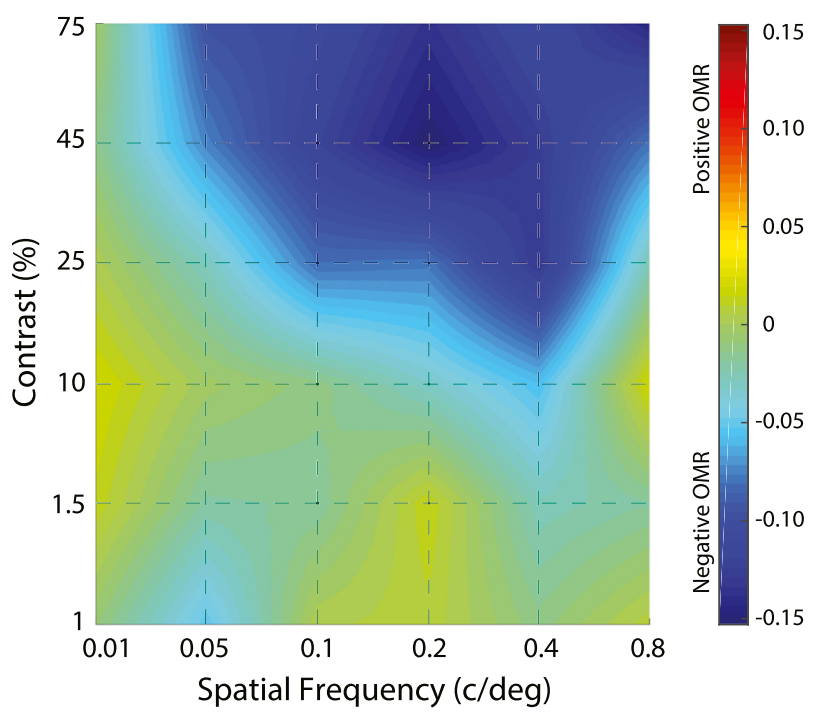

B
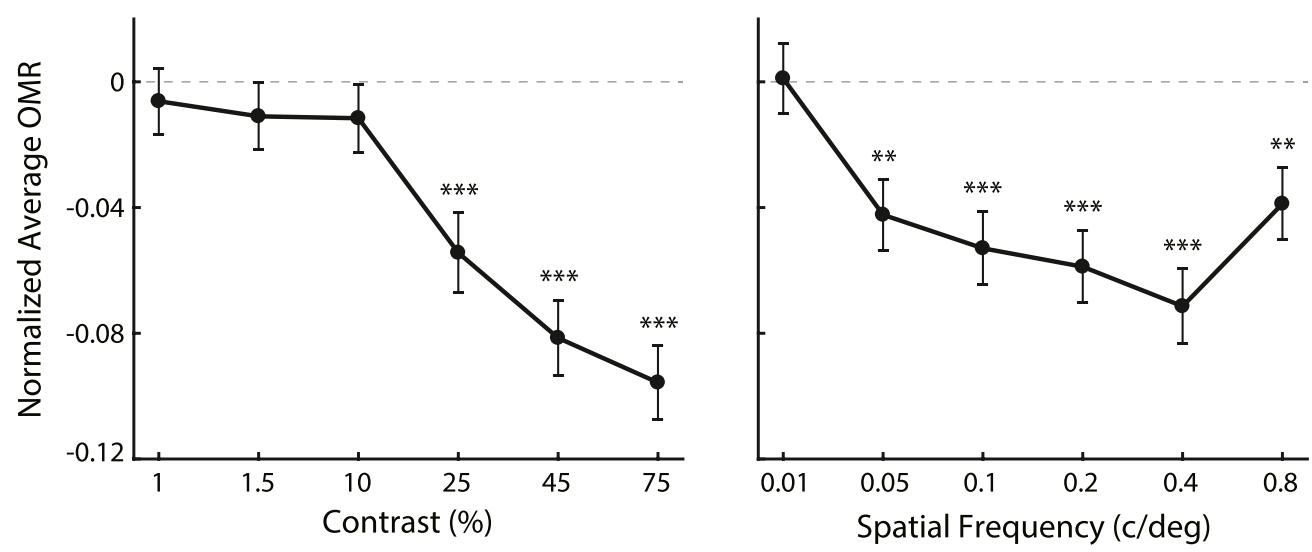

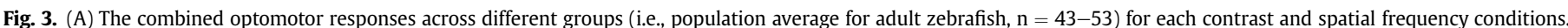

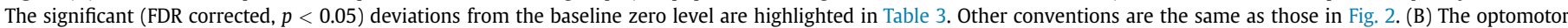

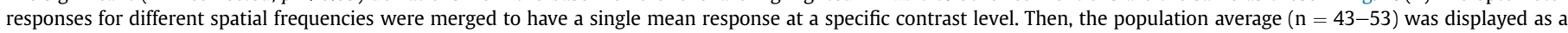

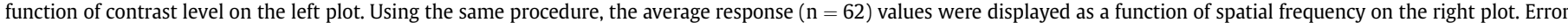

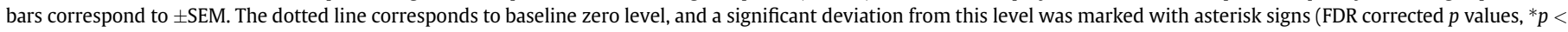
$0.05,{ }^{* *} p<0.01,{ }^{* * *} p<0.001$ ). The negative OMR became significant at contrast levels above $10 \%$ and/or spatial frequency values greater than $0.01 \mathrm{c} /$ deg. 
the responses such that higher contrast levels elicited stronger and more negative (opposite to the physical motion direction) OMR. This also demonstrates that the position shift of zebrafish increased as the visibility of motion was increased. Such dependency on contrast (Fig. 3B, left plot) has been typically reported by motion detection studies on humans and other vertebrates. In the spatial frequency domain, the strongest OMR was elicited by $0.4 \mathrm{c} / \mathrm{deg}$. Up to this spatial frequency, the negative OMR became stronger as the spatial frequency was increased. However, the negative OMR got weaker beyond this value, indicating that $0.4 \mathrm{c} / \mathrm{deg}$ spatial frequency was optimal to elicit an optomotor response. Given that OMR was significantly affected by visibility (i.e., contrast), this also suggests that zebrafish had the overall highest sensitivity to motion at this spatial frequency. This U-shaped dependency (Fig. 3B, right plot) is also consistent with the contrast sensitivity functions identified by previous OKR studies on adult zebrafish (Hollbach et al., 2015; Tappeiner et al., 2012). Using the merged data shown in Fig. 3, we also tested at each contrast and spatial frequency condition whether the elicited OMR was significantly different from zero baseline level (see also Supplementary Figure S4 for a separate representation of contrast and spatial frequency). We found significant OMRs at contrast levels above $10 \%$ (i.e., $25 \%, 45 \%$, and $75 \%$ ) and/or spatial frequency values greater than $0.01 \mathrm{c} / \mathrm{deg}(0.05,0.1$, $0.2,0.4$, and $0.8 \mathrm{c} / \mathrm{deg}$; see also Table 3 and Fig. $3 \mathrm{~B}$ ). The outcome of these additional tests revealed that the dependency of negative OMR on contrast level and spatial frequency is morphologically similar to those identified by previous motion studies. Moreover, they also provide the optimum combinations of contrast level and spatial frequency to elicit significant negative OMR in adult zebrafish.

To disentangle the sources of three-way interaction in the contrast domain, we combined all data across different spatial frequencies and performed additional post hoc comparisons on the merged data. As shown in Fig. 4, the wild-type young group had stronger and more negative OMR compared with the young aches $e^{\text {s55/+ }}$ mutants at $45 \%$ and $75 \%$ contrast levels. For the old groups, the overall effect of genotype at high contrast levels (e.g., $25 \%, 75 \%$ ) was in the opposite direction, and the ache $e^{s b 55 /+}$ group had relatively stronger OMR. Of note, the visual motion at these contrast levels elicited significant OMRs (Fig. 3B). Pairwise comparisons between different groups at each of the 6 contrast levels (Supplementary Table S3) indicated that the OMR of young ache $e^{\text {sb55/ }}$ ${ }^{+}$group at $75 \%$ contrast level was significantly different from those of old ache $e^{s 55 /+}$ mutants $(p=0.004$, Cliff's $\delta=0.53$ ) and young
A

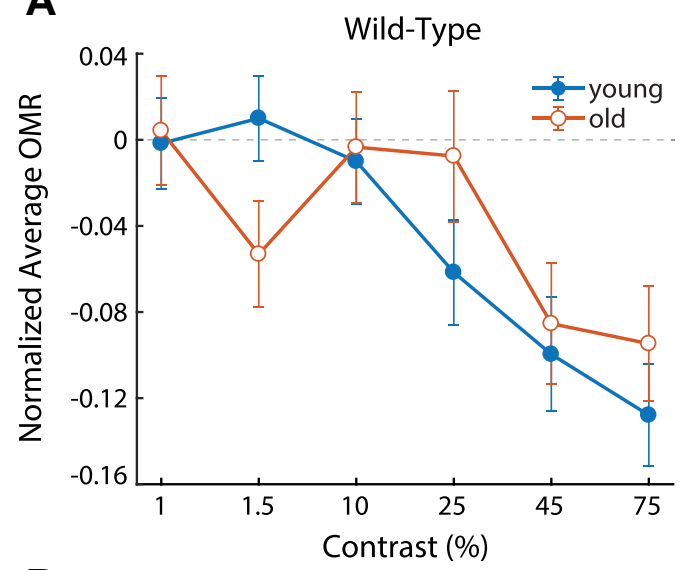

B

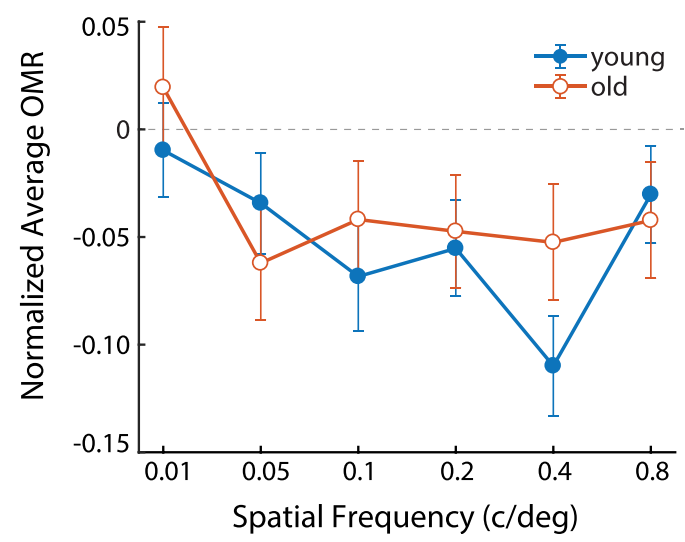

Ache
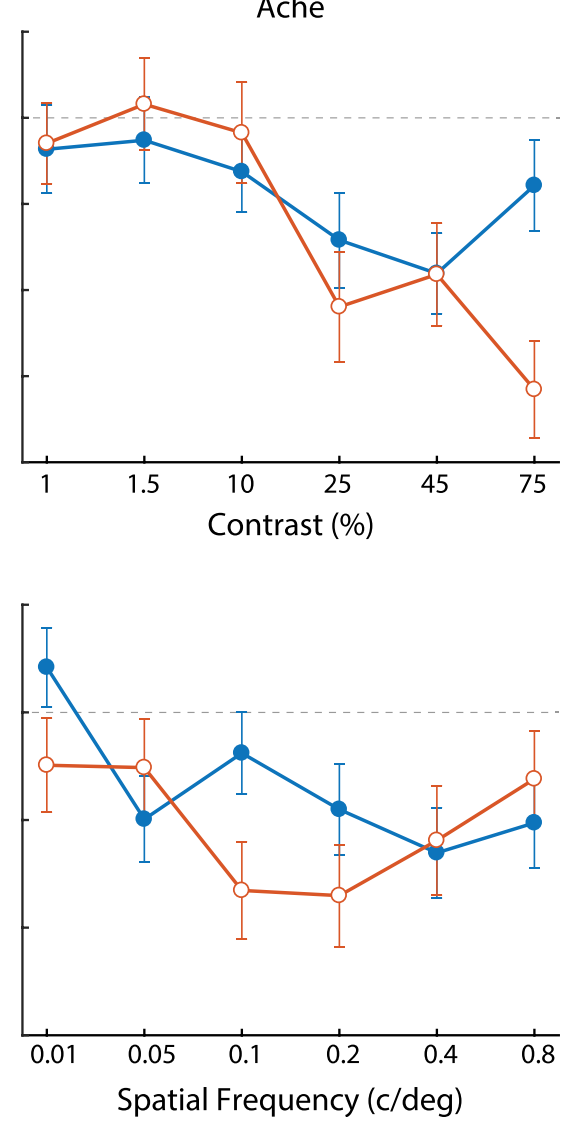

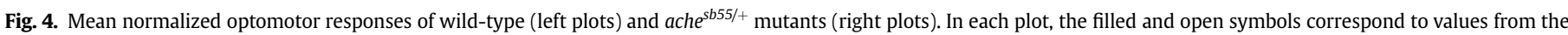

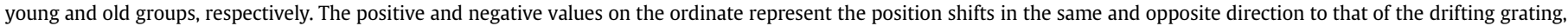

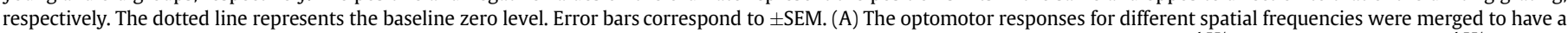

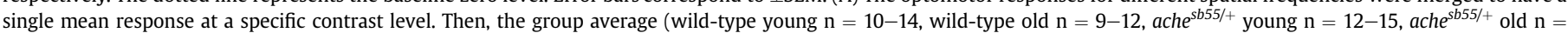

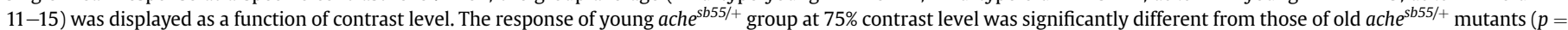

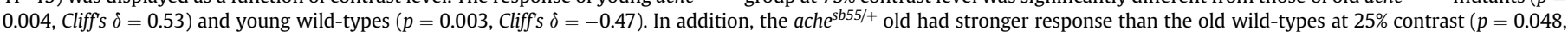

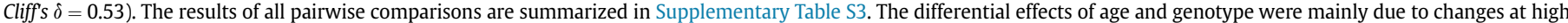

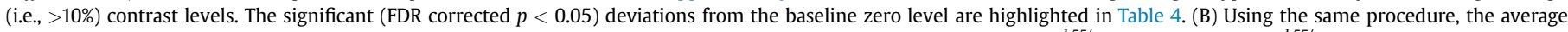
response values were displayed as a function of spatial frequency (wild-type young $\mathrm{n}=16$, wild-type old $\mathrm{n}=12$, ache $e^{\text {sb55/+ }}$ young $\mathrm{n}=19$, ache $e^{\text {sb55/+ }}$ old $\mathrm{n}=15$ ). 
wild-types ( $p=0.003$, Cliff's $\delta=-0.47$ ). In addition to this contrast level, the ache $e^{s b 55 /+}$ old had stronger OMR than the old wild-types at $25 \%$ contrast $(p=0.048$, Cliff's $\delta=0.53$ ). Comparing each contrast condition with the baseline zero level revealed that the negative OMR values at $45 \%$ and $75 \%$ contrast were significantly different than this level for both wild-type groups (Table 4). For the ache $e^{s b 5 /+}$ young group, only the $45 \%$ contrast level was significantly different. The OMR values of contrast above $10 \%$ percent were significantly different than the baseline level in the achesb55/+ old group, supporting the observation that this group exhibited stronger OMR. The outcome of these additional tests on the merged data suggests that the differential effects of age and genotype are mainly due to changes at high (i.e., $>10 \%$ ) contrast levels.

\section{Discussion}

In the present study, we investigated age-related changes in the motion detection and direction perception of wild-type and $a c h e^{s b 55 /+}$ zebrafish. Using zebrafish OMR to visual motion, we aimed to characterize these changes by systematically varying the contrast and spatial frequency of drifting gratings. In our measurements, we mainly found negative OMR (position shift in the opposite direction of visual motion) and the amount of this response was significantly affected by contrast and spatial frequency. More importantly, our analyses revealed a significant interaction between contrast, age (old vs. young), and genotype (wild-type vs. ache $e^{s b 55 /+}$ mutants). In other words, the effects of contrast on OMR values varied across different age and genotype groups. On the other hand, we did not find such interaction in the spatial frequency domain. In what follows, we provide the implications of these findings for zebrafish behavior, age-related changes in perception, and the functional links between the cholinergic system and visual motion processing.

\subsection{OMR of adult zebrafish}

The OMR has been observed in different animal species and described as the reflexive move of the animals to stabilize the displacement of the visual image on the retina. The zebrafish OMR has been mostly demonstrated using larvae (around 5-8 days postfertilization) and found to be in the direction of visual motion (i.e., positive OMR). On the other hand, research on adult zebrafish also pointed out a response in the opposite direction of visual motion (i.e., negative OMR). Using a drum that rotates clockwise/counterclockwise to generate optic flow, Maaswinkel and Li (2003) reported that the proportion of trials, in which the positive and negative OMRs of adult zebrafish is observed, depends on the speed, spatial and temporal frequencies of visual motion. Although these findings did not provide a relationship between the negative OMR magnitude and visual motion parameters (e.g., spatial frequency), they revealed

\section{Table 4}

One sample t-tests on the data shown in Fig. 4A to assess whether the OMR elicited by each condition were significantly different than the baseline zero level. The $p$ values for each contrast level are shown in separate columns. Significant $p$ values (FDR corrected, $p<0.05$ ) are highlighted in bold

\begin{tabular}{llllllr}
\hline Group & \multicolumn{6}{l}{ Contrast (\%) } \\
\cline { 2 - 7 } & 1 & 1.5 & 10 & 25 & 45 & \multicolumn{1}{c}{75} \\
\hline Wild-type & 0.949 & 0.828 & 0.828 & 0.102 & $\mathbf{0 . 0 0 6}$ & $<\mathbf{0 . 0 0 1}$ \\
Young & 0.896 & 0.070 & 0.896 & 0.896 & $\mathbf{0 . 0 1 5}$ & $\mathbf{0 . 0 1 5}$ \\
Old & & & & & & \\
ache ${ }^{\text {sb55/+ }}$ & & & & & \\
Young & 0.643 & 0.643 & 0.414 & 0.135 & $\mathbf{0 . 0 0 6}$ & 0.412 \\
Old & 0.783 & 0.783 & 0.783 & $\mathbf{0 . 0 1 0}$ & $\mathbf{0 . 0 0 9}$ & $<\mathbf{0 . 0 0 1}$ \\
\hline
\end{tabular}

that adult zebrafish can exhibit negative OMR on the significant number of trials. Relatively more comparable experimental design in terms of both visual stimulation and speed to the present study was used by Bak-Coleman et al. (2015). They systematically investigated OMR using different zebrafish groups. Interestingly, they showed that young larval zebrafish exhibit positive OMR, whereas adults show negative OMR. They hypothesized that the polarity reversal in OMR might be due to learned multisensory expectations. As the fish gets older, it might be better at differentiating exogenously exposed optic flow cues due to the experimental visual stimulation and the ones resulting from self-movement. For instance, when the fish swims in the same direction of visual motion, the visual cues are stabilized as if the fish is not moving. However, there is still a frontto-back water flow sensation detected by the lateral line system that signals forward motion, which contradicts the information coming from the visual system. They argue that the adult zebrafish might show negative OMR to overcome this contradiction by creating visual motion signals that are in the consistent direction (front-toback) with that of other nonvisual cues.

Although we observed positive OMR in some trials of specific conditions, our results highlight negative OMR to drifting gratings (see Supplementary Videos 1 and 3 for a sample of negative and positive OMR, respectively). In this respect, they confirm the previous findings on adult zebrafish. Our results provide further important and novel insights into the nature of negative OMR exhibited by adults. For instance, we found that the magnitude of negative OMR significantly depends on low-level visual parameters such as contrast and spatial frequency. A hypothesis based on learned multisensory expectations or alternative views should take into account these parameters and provide an explanation of why they significantly affect negative OMR. More importantly, the spatial frequency tuning curve of negative OMR revealed by our measurements are also in agreement with zebrafish contrast sensitivity functions. Using apparatus generating clockwise/counterclockwise moving gratings and optokinetic reflexive eye movements elicited by those gratings, previous research estimated the sensitivities of zebrafish for different spatial frequencies (Hollbach et al., 2015; Tappeiner et al., 2012). The contrast sensitivity functions were inverted U-shaped and peaked at an optimum frequency (typically around $0.1-0.2 \mathrm{c} / \mathrm{deg}$ ). Given that an increase in the visibility (contrast) leads to greater negative OMR, our findings suggest that the sensitivity peaks around $0.4 \mathrm{c} / \mathrm{deg}$ and declines when the spatial frequency was decreased or increased. In other words, this U-shaped spatial frequency dependency of negative OMR is qualitatively consistent with zebrafish sensitivity functions estimated with a different experimental design and another reflexive behavior. Overall, our findings highlight the dependency of negative OMR on low-level visual parameters and hence, the sensory aspect of negative OMR.

\subsection{Age and genotype interaction in the contrast domain}

There is a growing realization of 2 important models and perspectives for understanding age-related changes in perception. First, visual motion has been considered to be crucial for survival in a dynamic environment and hence, it has been extensively studied by vision scientists (Burr and Thompson, 2011; Kolers, 1972; Nakayama, 1985). A processing hierarchy and the motion systems operating at different stages of perceptual processing have been identified. Moreover, visual motion stimuli selectively engaging these systems have been designed. Therefore, previous motion studies provide an excellent conceptual framework to understand age-related changes at different stages of perceptual processing (Billino and Pilz, 2019). Second, the zebrafish has emerged as a promising model for understanding the cognitive and neurobiological changes during aging, as 
well as its use with potential anti-aging interventions (Adams and Kafaligonul, 2018). As mentioned in the previous sections, ache $e^{\text {sb55/ }}$ + mutants carrying a point mutation in the acetylcholinesterasecoding gene demonstrated a delayed aging phenotype in terms of behavioral measures such as entrainment to spatial-temporal cues and learning performance and flexibility, which are normally disrupted with normal aging in zebrafish (Yu et al., 2006). The present study uniquely combines these 2 emerging models and provides a comprehensive view on the effects of aging on perception. Particularly, this is to our knowledge the first systematic investigation on motion detection and perceived direction of adult zebrafish including both wild-type and ache $e^{\text {sb55/+ }}$ mutants.

Rather than an overall effect of aging, our results revealed a three-way interaction between contrast, genotype, and age. In other words, age-related differences in negative OMR values were largely dependent on stimulus parameters and zebrafish group. Ache $e^{\text {sb55/+ }}$ old group had stronger OMR at high contrast levels and hence suggesting an increased performance in the detection of motion direction. Interestingly, contrary to our expectations, we did not observe a similar improvement in the young group. In fact, the young ache $e^{s b 55 /+}$ group had smaller responses to visual motion at high contrast levels. A lack of improvement in the performance of young ache $e^{s b 55 /+}$ fish might be due to the altered course of development in their visual system. Besides slowing down cognitive aging (Yu et al., 2006), it is reasonable to hypothesize that this mutation may have also delayed the development of neural circuits essential to motion processing. Given that zebrafish larvae and adults elicit positive and negative OMR, respectively, the time frame of switching from positive OMR to negative OMR may be extended for young ache $e^{s 55 /+}$ mutants and thus they may not be mature enough to have a strong negative OMR to visual motion.

Visual motion has been extensively used to understand the effects of aging on human perception. Using different motion types, most of the early studies on humans pointed out overall age-related declines in the detection and discrimination of motion. It should be noted that there are also studies reporting no evidence of agerelated alterations in visual motion perception (see Billino and Pilz, 2019 for a review). Relatively recent findings suggested that the influence of age on motion perception is significantly dependent on the age groups, perceptual task, and stimulus parameters (Conlon et al., 2017; Pilz et al., 2017). That is to say, these findings highlight the differential effects of aging rather than an overall decline. By showing only a three-way interaction on optomotor responses, our behavioral results support this emerging view. Previous studies have indicated that cholinergic activation increases performance in various visual functions such as visual acuity (Kang et al., 2014), contrast sensitivity (Bhattacharyya et al., 2013), and motion direction discrimination (Thiele et al., 2012). Our findings suggest that the performance increase due to cholinergic activation is specific to certain stimulus parameters (i.e., contrast) and age groups. Although these findings are in agreement with the behavioral studies on humans reporting that cholinergic transmission plays an important role in motion processing (e.g., Kunchulia et al., 2019), they also emphasize that there is a complex relationship between visual stimulation and cholinergic system during neural aging. In fact, previous studies on the primary visual cortex showed the ACh level significantly increases response gain in the contrast domain. These modulations became dominant beyond the $10 \%$ contrast level (Disney et al., 2007; Soma et al., 2012). Of note, manipulation in the contrast level leads to a direct change in the motion energy and the signal to noise ratio of stimulation. Therefore, our behavioral findings highlighting the modulations in the contrast domain and particularly at high contrast levels ( $>10 \%)$ are in line with previous research and these properties of contrast level manipulation.
It is also worthwhile to mention that deteriorated visual acuity due to optical factors and alterations in retinal structure (Artal et al., 2003; Glasser et al., 1998; Loewenfeld, 1979) cannot simply explain the behavioral results presented here. Such an interpretation of data implies that there should be an overall age-related decline in the amplitude of negative OMR. However, as mentioned previously, we only observed changes in the contrast domain rather than spatial frequency. Moreover, these changes were restricted to high contrast levels and genotype-specific. Therefore, the significant three-way interaction on the behavioral measure cannot be due to just those low-level structural alterations.

\section{Conclusions}

To conclude, using a comprehensive view and experimental approach, we aimed to characterize the motion detection of adult zebrafish during aging. We found that adult zebrafish mainly exhibit a negative OMR to drifting gratings which is also significantly dependent on spatial frequency and contrast level. These findings highlight the dependency of negative optomotor responses on low-level visual parameters and hence, the sensory aspect of this response. Rather than an overall effect of aging, our results revealed a three-way interaction between contrast, genotype, and age. Therefore, they also point to a complex relationship between the physical characteristics of visual motion stimulation and the cholinergic system during neural aging. Overall, these behavioral results pave the way for a detailed investigation of the functional links between the physical motion characteristics and the cholinergic system in the zebrafish aging model which will ultimately have important implications for developing interventions to improve human visual performance during aging.

\section{Disclosure statement}

The authors have no actual or potential conflicts of interest.

\section{CRediT authorship contribution statement}

Aysenur Karaduman: Conceptualization, Data curation, Formal analysis, Investigation, Methodology, Resources, Validation, Visualization, Writing - original draft. Elif Tugce Karoglu-Eravsar: Methodology, Resources, Validation, Writing - review \& editing. Utku Kaya: Formal analysis, Methodology, Visualization, Writing - review \& editing. Alaz Aydin: Software, Visualization, Writing - review \& editing. Michelle M. Adams: Conceptualization, Resources, Supervision, Writing - review \& editing. Hulusi Kafaligonul: Conceptualization, Formal analysis, Methodology, Visualization, Funding acquisition, Supervision, Writing - review \& editing.

\section{Acknowledgments}

The authors thank Tulay Arayici and Mustafa Utkur for excellent technical assistance with animal experiments and İrem Hergüner for help during the video recording analyses. The authors are also grateful to Dr. Ayca Arslan-Ergul who was at the origin of this project.

Funding: This work was supported by the Scientific and Technological Research Council of Turkey (TUBITAK, grant number: 215S701). The authors Aysenur Karaduman and Elif T. Karoglu Eravsar were also supported by the National Scholarship Program for Ph.D. Students (TUBITAK 2211-E/A program).

Data availability statement: The original data set of this study is available at https://dx.doi.org/10.17632/9t59zjtx3p.1. 


\section{Appendix A Supplementary data}

Supplementary data to this article can be found online at https:// doi.org/10.1016/j.neurobiolaging.2020.10.018.

\section{References}

Adams, M.M., Kafaligonul, H., 2018. Zebrafish-A model organism for studying the neurobiological mechanisms underlying cognitive brain aging and use of potential interventions. Front. Cell Dev. Biol. 6, 135.

Adams, M.M., Shi, L., Linville, M.C., Forbes, M.E., Long, A.B., Bennett, C., Newton, I.G., Carter, C.S., Sonntag, W.E., Riddle, D.R., Brunso-Bechtold, J.K., 2008. Caloric restriction and age affect synaptic proteins in hippocampal CA3 and spatial learning ability. Exp. Neurol. 211, 141-149.

Ahmad, A., Spear, P.D., 1993. Effects of aging on the size, density, and number of rhesus monkey lateral geniculate neurons. J. Comp. Neurol. 334, 631-643.

Arslan-Ergul, A., Erbaba, B., Karoglu, E.T., Halim, D.O., Adams, M.M., 2016. Shortterm dietary restriction in old zebrafish changes cell senescence mechanisms. Neuroscience 334, 64-75.

Artal, P., Guirao, A., Berrio, E., Piers, P., Norrby, S., 2003. Optical aberrations and the aging eye. Int. Ophthalmol. Clin. 43, 63-77.

Avci, M.E., Keskus, A.G., Targen, S., Isilak, M.E., Ozturk, M., Atalay, R.C., Adams, M.M., Konu, O., 2018. Development of a novel zebrafish xenograft model in ache mutants using liver cancer cell lines. Sci. Rep. 8, 1570.

Bak-Coleman, J., Smith, D., Coombs, S., 2015. Going with, then against the flow: evidence against the optomotor hypothesis of fish rheotaxis. Anim. Behav. 107, $7-17$.

Ball, K., Sekuler, R., 1986. Improving visual perception in older observers. J. Gerontol. 41, 176-182.

Bartus, R., Dean, R., Beer, B., Lippa, A., 1982. The cholinergic hypothesis of geriatric memory dysfunction. Science 217, 408-414.

Behra, M., Cousin, X., Bertrand, C., Vonesch, J.-L., Biellmann, D., Chatonnet, A., Strähle, U., 2002. Acetylcholinesterase is required for neuronal and muscular development in the zebrafish embryo. Nat. Neurosci. 5, 111-118.

Benjamini, Y., Hochberg, Y., 1995. Controlling the false discovery rate: a practical and powerful approach to multiple testing. J. R. Stat. Soc. B 57, 289-300.

Benjamini, Y., Yekutieli, D., 2001. The control of the false discovery rate in multiple testing under dependency. Ann. Stat. 29, 1165-1188.

Bennett, P.J., Sekuler, R., Sekuler, A.B., 2007. The effects of aging on motion detection and direction identification. Vis. Res 47, 799-809.

Betts, L.R., Sekuler, A.B., Bennett, P.J., 2009. Spatial characteristics of center-surround antagonism in younger and older adults. J. Vis. 9, 25.

Bhattacharyya, A., Veit, J., Kretz, R., Bondar, I., Rainer, G., 2013. Basal forebrain activation controls contrast sensitivity in primary visual cortex. BMC Neurosci. $14,55$.

Biehl, S.C., Andersen, M., Waiter, G.D., Pilz, K.S., 2017. Neural changes related to motion processing in healthy aging. Neurobiol. Aging 57, 162-169.

Billino, J., Pilz, K.S., 2019. Motion perception as a model for perceptual aging. J. Vis. $19,3$.

Bilotta, J., Saszik, S., 2001. The zebrafish as a model visual system. Int. J. Devl Neurosci. 19, 621-629.

Boisgontier, M.P., Cheval, B., 2016. The anova to mixed model transition. Neurosci. Biobehav. Rev. 68, 1004-1005.

Bollmann, J.H., 2019. The zebrafish visual system: from circuits to behavior. Annu. Rev. Vis. Sci. 5, 269-293.

Born, R.T., Bradley, D.C., 2005. Structure and visual function of area MT. Annu. Rev. Neurosci. 28, 157-189.

Brainard, D.H., 1997. The psychophysics Toolbox. Spat. Vis. 10, 433-436.

Brauer, M., Curtin, J.J., 2018. Linear mixed-effects models and the analysis of nonindependent data: a unified framework to analyze categorical and continuous independent variables that vary within-subjects and/or within-items. Psychol. Methods 23, 389-411.

Burr, D., Thompson, P., 2011. Motion psychophysics: 1985-2010. Vis. Res. 51, 1431-1456.

Clayton, D.A., Mesches, M.H., Alvarez, E., Bickford, P.C., Browning, M.D., 2002. A hippocampal NR2B deficit can mimic age-related changes in long-term potentiation and spatial learning in the Fischer 344 rat. J. Neurosci. 22, 3628-3637.

Cohen, M.R., Maunsell, J.H.R., 2009. Attention improves performance primarily by reducing interneuronal correlations. Nat. Neurosci. 12, 1594-1600.

Conlon, E.G., Power, G.F., Hine, T.J., Rahaley, N., 2017. The impact of older age and sex on motion discrimination. Exp. Aging Res. 43, 55-79.

Disney, A.A., Aoki, C., Hawken, M.J., 2007. Gain modulation by nicotine in macaque V1. Neuron 56, 701-713.

Everitt, B.J., Robbins, T.W., 1997. Central cholinergic systems and cognition. Annu. Rev. Psychol. 48, 649-684.

Furey, M.L., Pietrini, P., Haxby, J.V., 2000. Cholinergic enhancement and increased selectivity of perceptual processing during working memory. Science 290, 2315-2319.

Gerhard, G.S., 2003. Comparative aspects of zebrafish (Danio rerio) as a model for aging research. Exp. Gerontol. 38, 1333-1341.

Glasser, A., Campbell, M.C.W., 1998. Presbyopia and the optical changes in the human crystalline lens with age. Vis. Res 38, 209-229.
Goard, M., Dan, Y., 2009. Basal forebrain activation enhances cortical coding of natural scenes. Nat. Neurosci. 12, 1444-1449.

Gori, S., Agrillo, C., Dadda, M., Bisazza, A., 2014. Do fish perceive illusory motion? Sci. Rep. 4, 6443.

Hasselmo, M.E., 1999. Neuromodulation: acetylcholine and memory consolidation. Trends Cogn. Sci. 3, 351-359.

Heck, R.H., Thomas, S.L., Tabata, L.N., 2014. Multilevel and longitudinal modeling with IBM SPSS. 2nd ed. Routledge, New York.

Herrero, J.L., Roberts, M.J., Delicato, L.S., Gieselmann, M.A., Dayan, P., Thiele, A., 2008 Acetylcholine contributes through muscarinic receptors to attentional modulation in V1. Nature 454, 1110-1114.

Hollbach, N., Tappeiner, C., Jazwinska, A., Enzmann, V., Tschopp, M., 2015. Photopic and scotopic spatiotemporal tuning of adult zebrafish vision. Front. Syst. Neurosci. 9, 20.

Howe, K., Clark, M.D., Torroja, C.F., Torrance, J., Berthelot, C., Muffato, M., Collins, J.E. Humphray, S., McLaren, K., Matthews, L., McLaren, S., Sealy, I., Caccamo, M., Churcher, C. Scott, C., Barrett, J.C., Koch, R., Rauch, G.J., White, S., Chow, W. Kilian, B., Quintais, L.T., Guerra-Assunção, J.A., Zhou, Y., Gu, Y., Yen, J., Vogel, J.H. Eyre, T., Redmond, S., Banerjee, R., Chi, J., Fu, B., Langley, E., Maguire, S.F. Laird, G.K., Lloyd, D., Kenyon, E., Donaldson, S., Sehra, H., Almeida-King, J., Loveland, J., Trevanion, S., Jones, M., Quail, M., Willey, D., Hunt, A., Burton, J., Sims, S., McLay, K., Plumb, B., Davis, J., Clee, C., Oliver, K., Clark, R., Riddle, C. Eliott, D., Threadgold, G., Harden, G., Ware, D., Mortimer, B., Kerry, G., Heath, P., Phillimore, B., Tracey, A., Corby, N., Dunn, M., Johnson, C., Wood, J., Clark, S. Pelan, S., Griffiths, G., Smith, M., Glithero, R., Howden, P., Barker, N., Stevens, C., Harley, J., Holt, K., Panagiotidis, G., Lovell, J., Beasley, H., Henderson, C., Gordon, D. Auger, K., Wright, D., Collins, J., Raisen, C., Dyer, L., Leung, K., Robertson, L., Ambridge, K., Leongamornlert, D., McGuire, S., Gilderthorp, R., Griffiths, C. Manthravadi, D., Nichol, S., Barker, G., Whitehead, S., Kay, M., Brown, J. Murnane, C., Gray, E., Humphries, M., Sycamore, N., Barker, D., Saunders, D., Wallis, J., Babbage, A., Hammond, S., Mashreghi-Mohammadi, M., Barr, L. Martin, S., Wray, P., Ellington, A., Matthews, N., Ellwood, M., Woodmansey, R., Clark, G., Cooper, J., Tromans, A., Grafham, D., Skuce, C., Pandian, R., Andrews, R. Harrison, E., Kimberley, A., Garnett, J., Fosker, N., Hall, R., Garner, P., Kelly, D. Bird, C., Palmer, S., Gehring, I., Berger, A., Dooley, C.M., Ersan-Ürün, Z., Eser, C. Geiger, H., Geisler, M., Karotki, L., Kirn, A., Konantz, J., Konantz, M., Oberländer, M. Rudolph-Geiger, S., Teucke, M., Osoegawa, K., Zhu, B., Rapp, A., Widaa, S. Langford, C., Yang, F., Carter, N.P., Harrow, J., Ning, Z., Herrero, J., Searle, S.M.J., Enright, A., Geisler, R., Plasterk, R.H.A., Lee, C., Westerfield, M., De Jong, P.J. Zon, L.I., Postlethwait, J.H., Nüsslein-Volhard, C., Hubbard, T.J.P., Crollius, H.R. Rogers, J., Stemple, D.L., 2013. The zebrafish reference genome sequence and its relationship to the human genome. Nature 496, 498-503.

Kalueff, A.V., Gebhardt, M., Stewart, A.M., Cachat, J.M., Brimmer, M., Chawla, J.S. Craddock, C., Kyzar, E.J., Roth, A., Landsman, S., Gaikwad, S., Robinson, K. Baatrup, E., Tierney, K., Shamchuk, A., Norton, W., Miller, N., Nicolson, T., Braubach, O., Gilman, C.P., Pittman, J., Rosemberg, D.B., Gerlai, R., Echevarria, D. Lamb, E., Neuhauss, S.C.F., Weng, W., Bally-Cuif, L., Schneider, H., 2013. Towards a comprehensive catalog of zebrafish behavior 1.0 and beyond. Zebrafish 10, 70-86.

Kalueff, A.V., Stewart, A.M., Gerlai, R., 2014. Zebrafish as an emerging model for studying complex brain disorders. Trends Pharmacol. Sci. 35, 63-75.

Kang, J.I., Groleau, M., Dotigny, F., Giguère, H., Vaucher, E., 2014. Visual training paired with electrical stimulation of the basal forebrain improves orientationselective visual acuity in the rat. Brain Struct. Funct. 219, 1493-1507.

Krauss, A., Neumeyer, C., 2003. Wavelength dependence of the optomotor response in zebrafish (Danio rerio). Vis. Res. 43, 1275-1284.

Kim, C.B.Y., Pier, L.P., Spear, P.D., 1997. Effects of aging on numbers and sizes of neurons in histochemically defined subregions of monkey striate cortex. Anat. Rec. 247, 119-128.

Kimmel, C.B., 1993. Patterning the brain of the zebrafish embryo. Annu. Rev. Neurosci. 16, 707-732.

Kishi, S., Bayliss, P.E., Uchiyama, J., Koshimizu, E., Qi, J., Nanjappa, P., Imamura, S. Islam, A., Neuberg, D., Amsterdam, A., Roberts, T.M., 2008. The identification of zebrafish mutants showing alterations in senescence-associated biomarkers. Plos Genet. 4, e1000152.

Kist, A.M., Portugues, R., 2019. Optomotor swimming in larval zebrafish is driven by global whole-field visual motion and local light-dark transitions. Cell Rep 29, 659-670.e3.

Kolers, P.A., 1972. Aspects of Motion Perception. Pergamon Press, Oxford.

Kunchulia, M., Kotaria, N., Pilz, K., Kotorashvili, A., Herzog, M.H., 2019. Associations between genetic variations and global motion perception. Exp. Brain Res. 237, 2729-2734.

Kretschmer, F., Kretschmer, V., Kunze, V.P., Kretzberg, J., 2013. OMR-arena: automated measurement and stimulation system to determine mouse visual thresholds based on optomotor responses. PLoS One 8, e78058.

Kretschmer, F., Tariq, M., Chatila, W., Wu, B., Badea, T.C., 2017. Comparison of optomotor and optokinetic reflexes in mice. J. Neurophysiol. 118, 300-316.

Langford, J., Koppel, S., 2006. The case for and against mandatory age-based assessment of older drivers. Transp. Res. Part F Traffic Psychol. Behav. 9 353-362.

Liang, Z., Yang, Y., Li, G., Zhang, J., Wang, Y., Zhou, Y., Leventhal, A.G., 2010. Aging affects the direction selectivity of MT cells in rhesus monkeys. Neurobiol. Aging $31,863-873$.

Loewenfeld, IE. 1979. Pupillary changes related to age. In: Thompson, S.H., Daroff, R., Frisèn, L., Glaser, J.S., Sanders, M.D. (Eds.), Topics in neuro-ophthalmology. Williams \& Wilkins, Baltimore, pp. 124-150. 
Lord, S.R., Smith, S.T., Menant, J.C., 2010. Vision and falls in older people: risk factors and intervention strategies. Clin. Geriatr. Med. 26, 569-581.

Maaswinkel, H., Li, L., 2003. Spatio-temporal frequency characteristics of the optomotor response in zebrafish. Vis. Res. 43, 21-30.

Morrison, J.H., Hof, P.R., 1997. Life and death of neurons in the aging brain. Science 278, 412-419.

Najafian, M., Alerasool, N., Moshtaghian, J., 2014. The effect of motion aftereffect on optomotor response in larva and adult zebrafish. Neurosci. Lett. 559, 179-183.

Nakayama, K., 1985. Biological image motion processing: a review. Vis. Res. 25, 625-660.

Naumann, E.A., Fitzgerald, J.E., Dunn, T.W., Rihel, J., Sompolinsky, H., Engert, F., 2016. From whole-brain data to functional circuit models: the zebrafish optomotor response. Cell 167, 947-960.

Norman, J.F., Ross, H.E., Hawkes, L.M., Long, J.R., 2003. Aging and the perception of speed. Perception 32, 85-96.

Orger, M.B., Baier, H., 2005. Channeling of red and green cone inputs to the zebrafish optomotor response, Vis, Neurosci. 22, 275-281.

Orger, M.B., de Polavieja, G.G., 2017. Zebrafish behavior: opportunities and challenges. Annu. Rev. Neurol. 40, 125-147.

Orger, M.B., Smear, M.C., Anstis, S.M., Baier, H., 2000. Perception of Fourier and nonFourier motion by larval zebrafish. Nat. Neurosci. 3, 1128-1133.

Owsley, C., 2016. Vision and aging. Annu. Rev. Vis. Sci. 2, 255-271.

Owsley, C., Ball, K., McGwin, G., Sloane, M.E., Roenker, D.L., White, M.F., Overley, E.T., 1998. Visual processing impairment and risk of motor vehicle crash among older adults. J. Am. Med. Assoc. 279, 1083-1088.

Pelli, D.G., 1997. The VideoToolbox software for visual psychophysics: transforming numbers into movies. Spat. Vis. 10, 437-442.

Pérez-Schuster, V., Kulkarni, A., Nouvian, M., Romano, S., Lygdas, K., Jouary, A., Dipoppa, M., Pietri, T., Haudrechy, M., Candat, V., Boulanger-Weill, J., Hakim, V., Sumbre, G., 2016. Sustained rhythmic brain activity underlies visual motion perception in zebrafish. Cell Rep. 17, 1098-1112.

Peters, A., Nigro, N.J., McNally, K.J., 1997. A further evaluation of the effect of age on striate cortex of the rhesus monkey. Neurobiol. Aging 18, 29-36.

Peters, A., Sethares, C., 1993. Aging and the Meynert cells in rhesus monkey primary visual cortex. Anat. Rec. 236, 721-729.

Pilz, K.S., Miller, L., Agnew, H.C., 2017. Motion coherence and direction discrimination in healthy aging. J. Vis. 17, 31.

Pix, W., Zanker, J.M., Zeil, J., 2000. The optomotor response and spatial resolution of the visual system in male Xenos vesparum (Strepsiptera). J. Exp. Biol. 203, 3397-3409.

Prusky, G.T., Alam, N.M., Beekman, S., Douglas, R.M., 2004. Rapid quantification of adult and developing mouse spatial vision using a virtual optomotor system. Invest. Ophthalmol. Vis. Sci. 45, 4611-4616.

Raghuram, A., Lakshminarayanan, V., Khanna, R., 2005. Psychophysical estimation of speed discrimination. II. Aging effects. J. Opt. Soc. Am. A. Opt. Image Sci. Vis. 22, 2269-2280.

Roberts, M.J., Zinke, W., Guo, K., Robertson, R., Mcdonald, J.S., Thiele, A., 2005. Acetylcholine dynamically controls spatial integration in marmoset primary visual cortex. J. Neurophysiol. 93, 2062-2072.

Rokem, A., Silver, M.A., 2013. The benefits of cholinergic enhancement during perceptual learning are long-lasting. Front. Comput. Neurosci. 7, 66.

Rokem, A., Silver, M.A., 2010. Cholinergic enhancement augments magnitude and specificity of visual perceptual learning in healthy humans. Curr. Biol. 20, 1723-1728.

Sarter, M., Hasselmo, M.E., Bruno, J.P., Givens, B., 2005. Unraveling the attentional functions of cortical cholinergic inputs: interactions between signal-driven and cognitive modulation of signal detection. Brain Res. Rev. 48, 98-111.

Schliebs, R., Arendt, T., 2011. The cholinergic system in aging and neuronal degeneration. Behav. Brain Res. 221, 555-563.

Schmitt, E.A., Dowling, J.E., 1999. Early retinal development in the zebrafish, Danio rerio: light and electron microscopic analyses. J. Comp. Neurol. 404, 515-536.
Schmolesky, M.T., Wang, Y., Pu, M., Leventhal, A.G., 2000. Degradation of stimulus selectivity of visual cortical cells in senescent rhesus monkeys. Nat. Neurosci. 3 384-390.

Schumann, C.M., Bloss, C.S., Barnes, C.C., Wideman, G.M., Carper, R. a, Pierce, K., Hagler, D., Schork, N., Lord, C., Courchesne, E., Akshoomoff, N., 2010. Longitudinal MRI study of cortical development through early childhood in autism. J. Neurosci. 30, 4419-4427.

Snowden, R.J., Kavanagh, E., 2006. Motion perception in the ageing visual system: minimum motion, motion coherence, and speed discrimination thresholds Perception 35, 9-24.

Soma, S., Shimegi, S., Osaki, H., Sato, H., 2012. Cholinergic modulation of response gain in the primary visual cortex of the macaque. J. Neurophysiol. 107, 283-291.

Soreq, H., Seidman, S., 2001. Acetylcholinesterase-new roles for an old actor. Nat. Rev. Neurosci. 2, 294-302.

Tabata, H., Shimizu, N., Wada, Y., Miura, K., Kawano, K., 2010. Initiation of the optokinetic response (OKR) in mice. J. Vis. 10, 13.

Tappeiner, C., Gerber, S., Enzmann, V., Balmer, J., Jazwinska, A., Tschopp, M., 2012 Visual acuity and contrast sensitivity of adult zebrafish. Front. Zool. 9, 1-6.

Terry, A.V., Buccafusco, J.J., 2003. The cholinergic hypothesis of age and Alzheimer's disease-related cognitive deficits: recent challenges and their implications for novel drug development. J. Pharmacol. Exp. Ther. 306, 821-827.

Thiele, A., Herrero, J.L., Distler, C., Hoffmann, K.-P., 2012. Contribution of cholinergic and GABAergic mechanisms to direction tuning, discriminability, response reliability, and neuronal rate correlations in macaque middle temporal area. J. Neurosci. 32, 16602-16615.

Thiele, A., Pooresmaeili, A., Delicato, L.S., Herrero, J.L., Roelfsema, P.R., 2009. Additive effects of attention and stimulus contrast in primary visual cortex. Cereb. Cortex 19, 2970-2981.

Vincent, S.L., Peters, A., Tigges, J., 1989. Effects of aging on the neurons within area 17 of rhesus monkey cerebral cortex. Anat. Rec. 223, 329-341.

Wang, K., Hinz, J., Zhang, Y., Thiele, T.R., Arrenberg, A.B., 2020. Parallel channels for motion feature extraction in the pretectum and tectum of larval zebrafish. Cell Rep. 30, 442-453.

Wang, Z., Yao, Z., Yuan, N., Liang, Z., Li, G., Zhou, Y., 2014. Declined contrast sensitivity of neurons along the visual pathway in aging cats. Front. Aging Neurosci. $6,163$.

Ward, L.M., Morison, G., Simmers, A.J., Shahani, U., 2018. Age-related changes in global motion coherence: conflicting haemodynamic and perceptual responses. Sci. Rep. 8, 10013.

Xu, Z., Cheng, X.E., 2017. Zebrafish tracking using convolutional neural networks. Sci. Rep. 7,1-11.

Yang, Y., Liang, Z., Li, G., Wang, Y., Zhou, Y., 2009a. Aging affects response variability of V1 and MT neurons in rhesus monkeys. Brain Res. 1274, 21-27.

Yang, Y., Liang, Z., Li, G., Wang, Y., Zhou, Y., Leventhal, A.G., 2008. Aging affects contrast response functions and adaptation of middle temporal visual area neurons in rhesus monkeys. Neuroscience 156, 748-757.

Yang, Y., Zhang, J., Liang, Z., Li, G., Wang, Y., Ma, Y., Zhou, Y., Leventhal, A.G., 2009b. Aging affects the neural representation of speed in macaque area MT. Cereb. Cortex 19, 1957-1967.

Yildizoglu, T., Riegler, C., Fitzgerald, J.E., Portugues, R., 2020. A neural representation of naturalistic motion-guided behavior in the zebrafish brain. Curr. Biol. 30 2321-2333.

Yu, L., Tucci, V., Kishi, S., Zhdanova, I.V., 2006. Cognitive aging in zebrafish. PLoS One 1 , e14.

Zeki, S., 2015. Area V5-a microcosm of the visual brain. Front. Integr. Neurosci. 9, 21.

Zhang, J., Wang, X., Wang, Y., Fu, Y., Liang, Z., Ma, Y., Leventhal, A.G., 2008. Spatial and temporal sensitivity degradation of primary visual cortical cells in senescent rhesus monkeys. Eur. J. Neurosci. 28, 201-207.

Zhdanova, I.V., Yu, L., Lopez-Patino, M., Shang, E., Kishi, S., Guelin, E., 2008. Aging of the circadian system in zebrafish and the effects of melatonin on sleep and cognitive performance. Brain Res. Bull. 75, 433-441. 